\title{
Influência de varióveis espirométricas e transporte mucociliar na funcionalidade de tabagistas leves
}

\section{Influence of spirometric variables and mucociliary transport on the functionality of light smokers}

\author{
Isis Grigoletto Silva'; Rebeca Nunes Silval; Diego Giulliano Destro Christofaro²; Ana Paula Coelho \\ Figueira Freire"; Dionei Ramos ${ }^{1}$; Ercy Mara Cipulo Ramos ${ }^{1}$ \\ 1 Departamento de Fisioterapia, Faculdade de Ciências e Tecnologia da Universidade Estadual Paulista "Júlio de Mesquita Filho" - \\ UNESP. Presidente Prudente, SP - Brasil. \\ 2 Departamento de Educação Física, Faculdade de Ciências e Tecnologia da Universidade Estadual Paulista "Júlio de Mesquita Filho" \\ - UNESP. Presidente Prudente, SP - Brasil. \\ Endereço para Correspondência: \\ Isis Grigoletto Silva \\ Rua Roberto Simonsen, 305 \\ 19060-900 - Presidente Prudente - SP [Brasil] \\ isis_grigoletto@hotmail.com
}

\begin{abstract}
Resumo
Introdução: $\mathrm{O}$ tabagismo provoca efeitos deletérios a saúde afetando o estado de saúde geral do indivíduo. Neste contexto, torna-se indispensável à avaliação da influência do fumo nas variáveis respiratórias e na capacidade funcional de tabagistas. Objetivo: Analisar a influência de variáveis espirométricas na capacidade de exercício de indivíduos tabagistas. Métodos: Estudo transversal com 78 indivíduos tabagistas, idade entre 40 e 60 anos, no qual foram avaliadas a função pulmonar (espirometria), a capacidade funcional (teste de caminhada de seis minutos-TC6) e a transportabilidade mucociliar nasal (teste do tempo de transporte da sacarina-TTS). Resultados: As variáveis espirométricas apresentaram correlação positiva significativa com o TC6 e o TTS não apresentou correlação com o TC6. Conclusão: A função pulmonar tem correlação com a capacidade funcional, no entanto o TTS não apresenta essa correlação em indivíduos tabagistas leves. Descritores: Tabagismo; Depuração Mucociliar; Espirometria.
\end{abstract}

\begin{abstract}
Introduction: Smoking causes deleterious health effects affecting the general health of the individual. In this context, it is essential to evaluate the influence of smoking on respiratory variables and on the functional capacity of smokers. Objective: To analyze the influence of spirometry variables on the exercise capacity of smokers. Methods: A cross-sectional study with 78 smokers, age between 40 and 60 years old, in which pulmonary function (spirometry), functional capacity (six-minute walk test - 6MWT) and nasal mucociliary transportability (Saccharin Transport Time Test - STT) were evaluated. Results: Spirometric variables had a significant postive correlation with the 6MWT and the STT did not present a correlation with the 6MWT. Conclusion: Pulmonary function correlated with functional capacity, however STT did not present this correlation in light smokers.
\end{abstract}

Keywords: Tobacco Use Disorder; Mucociliary Clearance; Spirometry. 


\section{Introdução}

O hábito tabagístico é considerado um dos principais fatores de risco para o desenvolvimento de doenças crônicas não transmissíveis ${ }^{1}$, como câncer e doenças respiratórias graves, e gera um gasto anual de meio trilhão de dólares para a saúde pública, além de ser responsável por aproximadamente 6 milhões de mortes ao ano ${ }^{2}$.

O uso do tabaco afeta o estado de saúde geral do indivíduo ${ }^{3}$, podendo causar alterações musculoesqueléticas e redução da capacidade cardiorrespiratória ${ }^{4}$, uma vez que o monóxido de carbono inalado na combustão do cigarro ${ }^{5}$ ificulta a oxigenação dos tecidos ${ }^{6}$. Diante disso, a literatura aponta que tabagistas apresentam redução da capacidade de exercício quando comparados a não tabagistas, e que esta possui correlação com o nível de dependência à nicotina ${ }^{7}$

A fumaça do cigarro apresenta radicais e oxidantes prejudiciais aos músculos esqueléticos, que levam a atrofia de fibras musculares do tipo I e ao aumento da atividade glicolítica ${ }^{8}$, influenciando de forma negativa na capacidade funcional (CF), visto que as disfunções musculoesqueléticas, bem como os marcadores próinflamatórios, levam a redução da capacidade de exercício nestes indivíduos ${ }^{8}$. Portanto, a avaliação da CF é de extrema importância para verificar a capacidade do indivíduo em realizar suas atividades no dia a dia ${ }^{9} \mathrm{e}$, para isto, podem ser utilizados testes de acordo com a disponibilidade de recursos ou de acordo com as limitações dos pacientes. Dentre tais testes podemos citar o Shuttle Walking Test (SWT) e o Teste de Caminhada de Seis Minutos (TC6) ${ }^{9}$, sendo o segundo uma ferramenta capaz de avaliar a CF do indivíduo, apresentando de moderada a forte correlação com o teste de consumo máximo de oxigênio ${ }^{10}$.

Estudos demonstram que a eficiência do transporte mucociliar (TMC), principal mecanismo de defesa das vias aéreas ${ }^{11}$, também apresenta-se alterada em tabagistas ${ }^{12}$. Tal fato pode estar relacionado a inalação das mais de
7000 substâncias tóxicas presentes na fumaça do cigarro $^{13}$, uma vez que as alterações no TMC podem ocorrer em diversas condições, dentre elas, frente a exposição de partículas nocivas ${ }^{14}$. O Teste de Trânsito da Sacarina (TTS), permite avaliação da integridade do transporte mucociliar, este teste apresenta baixo custo, fácil aplicação e análise, além disso apresenta boa correlação com a atividade mucociliar traqueobrônquica, demonstrando assim se o paciente apresenta alterações no transporte mucociliar decorrente do tabagismo ${ }^{15}$.

Ainda, o tabagismo gera um rápido declínio da função pulmonar (FP). Neste contexto, evidências mostram que o hábito de fumar causa redução das variáveis espirométricas ${ }^{16}$, inclusive ao consumir cigarros com baixas doses de nicotina ${ }^{17}$.

Diante do exposto, sabe-se que a CF e a FP de tabagistas podem estar comprometidas, causando impacto direto na qualidade de vida ${ }^{3}$.

Dessa forma, o conhecimento de tais alterações por parte dos tabagistas promove maior embasamento para o alerta e tratamento do tabagismo. Diante disto, é indispensável a avaliação da influência do uso crônico do cigarro nestas variáveis, a fim de contribuir para uma melhor elucidação sobre os efeitos deletérios em tabagistas aparentemente saudáveis, e para que, assim, profissionais da saúde possam disseminar informações a respeito da necessidade eminente da cessação do tabagismo. Portanto, o objetivo do presente estudo foi verificar a influência das variáveis espirométricas na capacidade de exercício de indivíduos tabagistas, hipotetizando-se, portanto, que a capacidade de exercício possua correlação com as variáveis espirométricas, bem como com a transportabilidade mucociliar em indivíduos tabagistas.-

\section{Materiais e métodos}

\section{Casuística}

Este foi um estudo observacional do tipo transversal com amostra por conveniência, no 
qual 78 homens e mulheres tabagistas, com faixa etária entre 40 e 60 anos de idade foram avaliados. Todos os participantes faziam parte do programa de cessação tabagística denominado Programa de Orientação e Conscientização Antitabagismo (PROCAT) da Faculdade de Ciências e Tecnologia FCT/UNESP localizada em Presidente Prudente - SP ${ }^{18}$.

Não foram incluídos no estudo indivíduos que apresentavam desvio de septo nasal, déficits neurológicos, neuromusculares, cardiovasculares, musculoesqueléticos e cognitivos que pudessem interferir nas avaliações, bem como pacientes sem colaboração frente ao protocolo da pesquisa, além do não comparecimento aos dias de avaliação.

Os voluntários foram previamente comunicados sobre os objetivos e procedimentos do estudo, e após concordarem e assinarem o termo de consentimento livre e esclarecido, passaram a fazer parte efetiva da pesquisa. Esta pesquisa foi submetida à apreciação do Comitê de Ética da FCT/UNESP de Presidente Prudente e aprovada pelo mesmo (processo $n^{\circ} 18 / 2011$ ) e todos os procedimentos seguiram a Resolução 466/2012 do Conselho Nacional de Saúde. O fluxograma de perdas do estudo pode ser observado na figura 1.

\section{Protocolo experimental}

O protocolo foi realizado no Centro de Estudos e de Atendimentos em Fisioterapia e Reabilitação (CEAFIR) na FCT/UNESP de Presidente Prudente. No primeiro dia de avaliação os pacientes participaram de uma entrevista, onde foram obtidos dados pessoais (nome, idade e sexo) e histórico tabagístico (número de cigarros/dia e índice anos/maço). Os voluntários responderam ainda ao Teste de Dependência à Nicotina de Fagerstrom, capaz de mensurar o nível de dependência à nicotina. Neste mesmo dia, foram verificados os dados antropométricos (peso e altura) e, em seguida, os indivíduos foram avaliados quanto a FP por meio da espirometria. No segundo dia de avaliação foi realizado o teste do Tempo de Trânsito da Sacarina (TTS) e, em seguida, o Teste de Caminhada de Seis Minutos (TC6). Os indivíduos foram orientados a não fazer uso de medicamentos tais como anestésicos, analgésicos barbitúricos, calmantes e antidepressivos, bem como bebidas alcoólicas e substâncias a base de cafeína no período mínimo de 12 horas antes da mensuração do TTS. Os testes foram realizados em ambiente com temperatura $\left(24^{\circ} \mathrm{C}\right)$ e humidade relativa do ar (50 a 60\%) controladas, no período da manhã (das 08 horas às 09 horas).

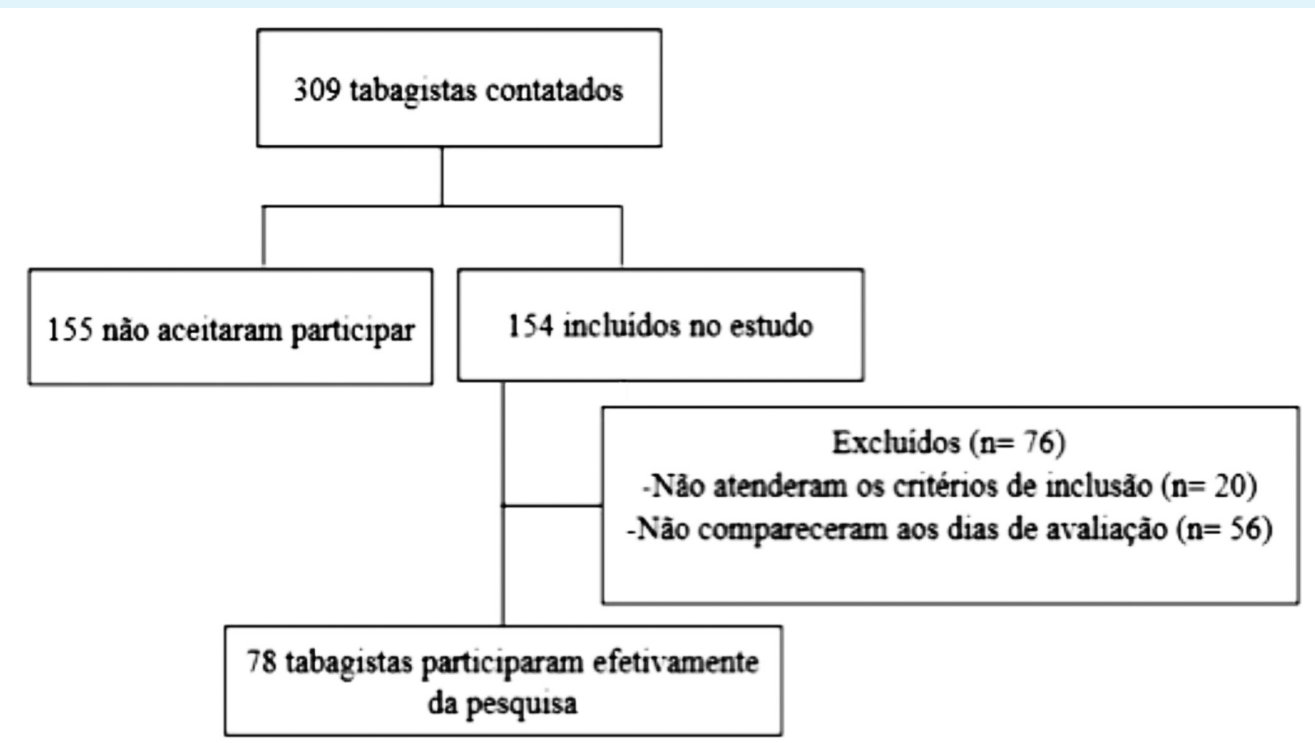

Figura 1: Fluxograma de perdas do estudo

Fonte: Arquivo dos autores. 


\section{Prova de função pulmonar}

As medidas de função pulmonar foram obtidas de todos os indivíduos por meio da espirometria, realizada e analisada de acordo com as diretrizes da American Thoracic Society e European Respiratory Society ${ }^{19}$. Para isso, utilizou-se espirômetro portátil (Spirobank-MIR, Itália, versão 3.6). Foram obtidos os valores de $\mathrm{VEF}_{1}$ (Volume Expiratório Forçado no primeiro segundo), CVF (Capacidade Vital Forçada) e $\mathrm{VEF}_{1} / \mathrm{CVF}$ (Relação entre os dois índices). Os valores de referência utilizados foram específicos para a população brasileira ${ }^{20}$.

\section{Teste de caminhada de seis minutos (TC6)}

O teste de caminhada de seis minutos (TC6) foi utilizado para avaliar a capacidade funcional dos participantes, e foi realizado de acordo com as diretrizes da American Thoracic Society ${ }^{9}$. Os valores experimentais obtidos foram referenciados aos valores normais fornecidos por Enright e Sherrill ${ }^{21}$.

\section{Teste do tempo de trônsito da sacorina (TTS)}

A avaliação do transporte mucociliar foi realizada por meio do Teste do Tempo de Trânsito da Sacarina (TTS). Para tanto, os participantes foram posicionados sentados com a cabeça estendida a $10^{\circ}$. O teste foi iniciado pela introdução de aproximadamente 250 microgramas de sacarina sódica granulada por meio de um canudo plástico, sob controle visual, a aproximadamente dois centímetros para dentro da narina direita. A partir deste momento, o cronômetro foi acionado e os indivíduos foram orientados a não andar, não falar, não tossir, não espirrar, não coçar ou assoar o nariz, além de serem instruídos a engolir poucas vezes por minuto até que sentissem um sabor em sua boca, então o examinador foi imediatamente avisado por meio de um gesto do avaliado e o tempo foi registrado ${ }^{22}$.

\section{Anólise Estatística}

Para a análise dos dados foi utilizado o programa estatístico SPSS versão 23.0. A relação entre a capacidade funcional (TC6) e as variáveis espirométricas $\left(\mathrm{VEF}_{1}, \mathrm{CVF}, \mathrm{VEF}_{1} / \mathrm{CVF}\right.$ e TTS) foi realizada pela correlação de Spearman. Os dados foram apresentados em mediana e intervalo interquartílico de $25-75 \%$ devido a sua distribuição. Para o gráfico da análise de correlação foi utilizado o programa MedCalc. A significância estatística utilizada foi pré-estabelecida em $5 \%$.

\section{Resultados}

Setenta e oito indivíduos tabagistas foram incluídos no estudo. A caracterização da amostra pode ser observada na Tabela 1 , na qual os dados foram expressos em mediana e intervalo interquartilico de $25-75 \%$.

A proporção de homens foi maior do que a de mulheres. Os voluntários apresentaram carga tabagística leve ${ }^{23}(5,695 \pm 1,545$ anos/maço) e moderada dependência a nicotina $(5,731 \pm 2,166$ pontos) segundo o teste de Fagerström ${ }^{24}$. As variáveis $\mathrm{VEF}_{1}$ absoluta e CVF absoluta obtidas por meio da espirometria se apresentaram normais segundo os valores preditos por Pereira et al. para a população brasileira ${ }^{20}$. Os valores de TTS foram considerados normais (valor de normalidade: $12,3 \pm 3,4 \mathrm{~min})^{22}$, o que indica função mucociliar preservada. No TC6, o valor percorrido se apresentou próximo ao predito para a amostra $(99,47 \% \text { predito })^{21}$, dessa forma, os valores de distância percorrida atingidos no TC6 podem ser considerados normais. Na tabela 2 é possível observar a análise de correlação entre as variáveis respiratórias e o TC6 e, na figura 2, o gráfico da análise de correlação destas mesmas variáveis.

\section{Discussão}

No presente estudo foi verificado que a $\mathrm{CF}$ apresenta correlação positiva com a FP de indi- 

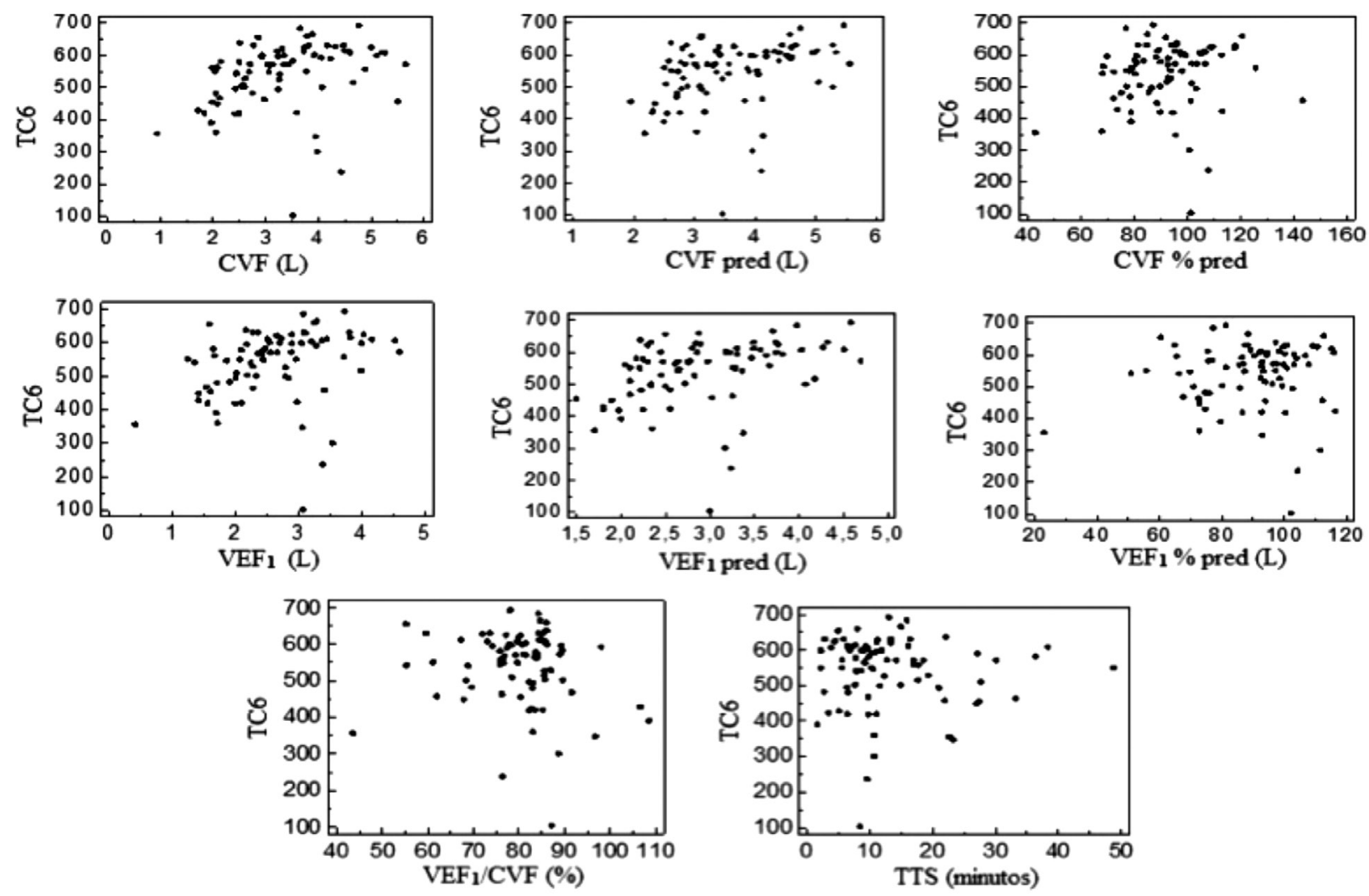

Figura 2: Grófico da análise de correlação entre varióveis espirométricas (CVF: capacidade vital forçada em litros; $\mathrm{VEF}_{1}$ : volume expiratório forçado no primeiro segundo em litros; $\mathrm{VEF}_{1} /$ CVF: relação entre o VEF 1 e CVF em porcentagem) e entre o TTS: teste do tempo de trânsito da sacarina em minutos com o Teste de Caminhada de seis minutos (TC6)

Fonte: Arquivo dos autores.

sido suficiente para promover danos estruturais significativos capazes de reduzir o TTS, o que está de acordo com os achados de Proença et. al em $2012^{29}$ e com Xavier et. al em $2013^{30}$, que afirmaram existir deficiência no TMC em fumantes com carga tabagística moderada e alta quando comparados com fumantes leves ${ }^{29,30}$.

No que diz respeito às implicações clínicas, o presente estudo destaca que as variáveis analisadas interferem de forma importante na qualidade de vida e no cotidiano de tabagistas $^{3}$, sendo necessário o entendimento de tais prejuízos, para que a população tabagista seja esclarecida sobre os malefícios do cigarro, mesmo quando a exposição ao fumo ainda não foi suficiente para causar distúrbios pulmonares ou redução da CF.
Como limitações, considera-se o fato de um maior número de indivíduos tabagistas do sexo masculino terem sido incluídos no estudo, o tamanho amostral reduzido e o fato de não ter sido realizada a análise estratificada da amostra de acordo com a carga tabagística, o que poderia revelar achados interessantes, uma vez que a este dado pode influenciar nas variáveis primárias desse estudo. Além disso a não avaliação do consumo máximo de oxigênio destes indivíduos devido a necessidade de equipamento adequado bem como devido ao seu custo elevado e a necessidade de equipe especializada neste tipo de avaliação, pode ser apontada como outra limitação do presente estudo. Neste contexto, estudos futuros devem ser compostos por amostras maiores, que permitam analisar e comparar grupos com diferentes cargas ta- 
bagísticas, o que não foi possível no presente estudo devido ao número amostral reduzido, bem como sugere-se também que estudos futuros realizados com tabagistas leves avaliem o consumo máximo de oxigênio destes indivíduos por meio de teste específico.

\section{Conclusões}

Tabagistas com carga tabagística leve apresentam relação entre a capacidade funcional e a função pulmonar, no entanto, não há influência da transportabilidade mucociliar na capacidade funcional destes indivíduos.

\section{Agradecimentos}

Os autores gostariam de agradecer a todos os voluntários pela participação no presente estudo e a UNESP por ter cedido o espaço para a realização deste. Além disso, gostaríamos de agradecer ao Programa de Orientação e Conscientização Antitabagismo e à Pró-Reitoria de Extensão Universitária pelo apoio financeiro.

Os autores declaram não haver conflitos de interesse.

\section{Referências}

1. Lim SS, Vos T, Flaxman AD, Danaei G, Shibuya

$\mathrm{K}$, Adair-Rohani $\mathrm{H}$, et al. A comparative risk assessment of burden of disease and injury attributable to 67 risk factors and risk factor clusters in 21 regions, 1990-2010: a systematic analysis for the Global Burden of Disease Study 2010. J. Lancet. 2012;380(9859):2224-60.

2. Organization $\mathrm{WH}$. WHO report on the global tobacco epidemic: enforcing bans on tobacco advertising, promotion and sponsorship. Geneva, Switzerland. 2013.

3. E N. Consumo de tabaco. Efeitos na saúde. RPMGF. 2006;22(2).
4. Kobayashi Y, Takeuchi T, Hosoi T, Loeppky JA. Effects of habitual smoking on cardiorespiratory responses to sub-maximal exercise. J. physiol. anthropol. appl. human sci. 2004;23(5):163-9.

5. Kalay N, Ozdogru I, Cetinkaya Y, Eryol NK, Dogan A, Gul I, et al. Cardiovascular effects of carbon monoxide poisoning. Am. j. cardiol. 2007;99(3):322-4.

6. Blumenthal I. Carbon monoxide poisoning. J. R. Soc. Med. 2001;94(6):270-2.

7. Lee CL, Chang WD. The effects of cigarette smoking on aerobic and anaerobic capacity and heart rate variability among female university students. Int. j. womens. health. 2013;5:667-79.

8. Montes de Oca M, Loeb E, Torres SH, De Sanctis J, Hernandez N, Talamo C. Peripheral muscle alterations in non-COPD smokers. Chest. 2008;133(1):13-8.

9. Brooks D, Solway S, Gibbons WJ. ATS statement on six-minute walk test. Am. j. respir. crit. care. med. 2003;167(9):1287.

10. Vagaggini B, Taccola M, Severino S, Marcello M, Antonelli S, Brogi S, et al. Shuttle walking test and 6-minute walking test induce a similar cardiorespiratory performance in patients recovering from an acute exacerbation of chronic obstructive pulmonary disease. Respiration; 2003;70(6):579-84

11. Chodzko-Zajko WJ, Proctor DN, Fiatarone Singh MA, Minson CT, Nigg CR, Salem GJ, et al. American College of Sports Medicine position stand. Exercise and physical activity for older adults. Med. sci. sports exerc. 2009;41(7):1510-30.

12. Cohen NA, Zhang S, Sharp DB, Tamashiro E, Chen B, Sorscher EJ, et al. Cigarette smoke condensate inhibits transepithelial chloride transport and ciliary beat frequency. Laryngoscope. 2009;119(11):2269-74.

13. National Center for Chronic Disease P, Health Promotion Office on S, Health. Reports of the Surgeon General. The Health Consequences of Smoking-50 Years of Progress: A Report of the Surgeon General. Atlanta (GA): Centers for Disease Control and Prevention (US); 2014.

14. Elliott MK, Sisson JH, Wyatt TA. Effects of cigarette smoke and alcohol on ciliated tracheal epithelium and inflammatory cell recruitment. Am. j. respir. cell mol. biol. 2007;36(4):452-9. 
15. Trindade SH, de Mello JF, Jr., Mion Ode G, LorenziFilho G, Macchione M, Guimaraes ET, et al. Methods for studying mucociliary transport. Braz J Otorhinolaryngol. 2007;73(5):704-12.

16. Salepci B, Caglayan B, Fidan A, Parmaksiz ET, Kiral N, Comert SS, et al. The Effect of Pulmonary Function Testing on the Success of Smoking Cessation. Respir. care. 2016;61(8):1073-80.

17. Ferrari M, Zanasi A, Nardi E, Morselli Labate AM, Ceriana P, Balestrino A, et al. Short-term effects of a nicotine-free e-cigarette compared to a traditional cigarette in smokers and non-smokers. BMC pulm. med. 2015;15:120.

18. Freire APCFR, Ramos D, Silva BSA, David RM, Pestana PRS, Fernandes RA, et al. Resultados de um programa de cessação tabagística: análise de novos procedimentos / Results of smoking cessation program: analysis of new procedures. ConsSaude. 2014;13(3).

19. Miller MR, Hankinson J, Brusasco V, Burgos F, Casaburi R, Coates A, et al. Standardisation of spirometry. Eur. respir. j. 2005;26(2):319-38.

20. Duarte AA, Pereira CA, Rodrigues SC. Validation of new brazilian predicted values for forced spirometry in caucasians and comparison with predicted values obtained using other reference equations. J. bras. pneumol. 2007;33(5):527-35.

21. Enright PL, Sherrill DL. Reference equations for the six-minute walk in healthy adults. Am. j. respir. crit. care med. 1998;158(51):1384-7.

22. Salah B, Dinh Xuan AT, Fouilladieu JL, Lockhart A, Regnard J. Nasal mucociliary transport in healthy subjects is slower when breathing dry air. Eu. respir. j. 1988;1(9):852-5

23. Kondo T, Osugi S, Shimokata K, Honjo H, Morita Y, Maeda $\mathrm{K}$, et al. Smoking and smoking cessation in relation to all-cause mortality and cardiovascular events in 25,464 healthy male Japanese workers. Circ J. 2011;75(12):2885-92.
24. Carmo JTdP, Antonio Andrés. A adaptaçäo ao português do Fagerström test for nicotine dependence (FTND) para avaliar a dependência e tolerância à nicotina em fumantes brasileiros / Adaptation into portuguese for the Fagerström test for nicotine dependence (FTND) to evaluate the dependence and tolerance for nicotine in brazilian smokers. RBM rev. bra. med. 2002;59(1/2):73-80.

25. Rodrigues SL MH, Viegas CA. Teste de caminhada de seis minutos: estudo do efeito do aprendizado em portadores de doença pulmonar obstrutiva crônica. J. pneumol. 2004;30(2):121-25.

26. Mesquita R, Goncalves CG, Hayashi D, Costa Vde S, Teixeira Dde C, de Freitas ER, et al. Smoking status and its relationship with exercise capacity, physical activity in daily life and quality of life in physically independent, elderly individuals. Physiotherapy. 2015;101(1):55-61.

27. Lee G, Walser TC, Dubinett SM. Chronic inflammation, chronic obstructive pulmonary disease, and lung cancer. Curr. opin. pulm. med. 2009;15(4):303-7.

28. Utsugi H, Nakamura H, Suzuki T, Maeno T, Nagata M, Kanazawa M. Associations of lifelong cigarette consumption and hypertension with airflow limitation in primary care clinic outpatients in Japan. Respir Investig. 2016;54(1):35-43.

29. Proenca M, Pitta F, Kovelis D, Mantoani LC, Furlanetto KC, Zabatiero J, et al. Mucociliary clearance and its relation with the level of physical activity in daily life in healthy smokers and nonsmokers. Rev. port. pneumol. 2012;18(5):233-8.

30. Xavier RF, Ramos D, Ito JT, Rodrigues FM, Bertolini GN, Macchione M, et al. Effects of cigarette smoking intensity on the mucociliary clearance of active smokers. Respiration. 2013;86(6):479-85. 